\title{
The Relationship Between Resilience and Sleep Quality During the Second Wave of the COVID-I 9 Pandemic: A Longitudinal Study
}

\author{
Vittorio Lenzo iD ' \\ Alberto Sardella ${ }^{2}$ \\ Alessandro Musetti (iD) 3 \\ Maria Francesca Freda ${ }^{4}$ \\ Daniela Lemmo ${ }^{4}$ \\ Elena Vegni ${ }^{5}$ \\ Lidia Borghi ${ }^{5}$ \\ Giuseppe Plazzi ${ }^{6,7}$ \\ Laura Palagini ${ }^{8,9}$ \\ Gianluca Castelnuovo ${ }^{10,11}$ \\ Roberto Cattivelli ${ }^{12}$ \\ Rachele Mariani ${ }^{13}$ \\ Giovanni Michelini ${ }^{14}$ \\ Tommaso Manari (iD ${ }^{3}$ \\ Emanuela Saita ${ }^{10}$ \\ Maria C Quattropani (iD) 15 \\ Christian Franceschini iD ${ }^{16}$
}

'Department of Social and Educational Sciences of the Mediterranean Area, University for Foreigners "Dante Alighieri” of Reggio Calabria, Reggio, Calabria, Italy; ${ }^{2}$ Department of Clinical and Experimental Medicine, University of Messina, Messina, Italy; ${ }^{3}$ Department of Humanities, Social Sciences and Cultural Industries, University of Parma, Parma, Italy; ${ }^{4}$ Department of Humanistic Studies, University of Naples Federico II, Naples, Italy; ${ }^{5}$ Department of Health Sciences, University of Milan, Milan, Italy; ${ }^{6}$ Department of Biomedical, Metabolic and Neural Sciences, University of Modena and Reggio Emilia, Modena, Italy; 'IRCCS Istituto delle Scienze Neurologiche di Bologna, Bologna, Italy; ${ }^{8}$ Department of Clinical and Experimental Medicine, Psychiatric Section, University of Pisa, Azienda Ospedaliera Universitaria Pisana (AUOP), Pisa, Italy; ${ }^{9}$ Department of Neuroscience and Rehabilitation, University of Ferrara, Ferrara, Italy; ${ }^{10}$ Department of Psychology, Catholic University of Milan, Milan, Italy; "Istituto Auxologico Italiano IRCCS, Psychology Research Laboratory, Ospedale San Giuseppe, Verbania, Italy; ${ }^{2}$ Department of Psychology, University of Bologna, Bologna, Italy; ${ }^{13}$ Department of Dynamic and Clinical Psychology, University of Rome "La Sapienza", Roma, Italy; ${ }^{14}$ Sigmund Freud University, Milano, Italy; ${ }^{15}$ Department of Educational Sciences, University of Catania, Catania, Italy; ${ }^{16}$ Department of Medicine and Surgery, University of Parma, Parma, Italy

Correspondence: Vittorio Lenzo

Department of Social and Educational Sciences of the Mediterranean Area, University for Foreigners "Dante Alighieri" of Reggio Calabria Via del Torrione 95, Reggio Calabria, 89125, Italy

Email v.lenzo@unidarc.it
Purpose: Previous studies showed poor sleep quality during the first Italian lockdown consequent to the quick spread of the virus. Poor sleep quality remained stable during the so-called "second wave", which started in Autumn 2020. This study aimed to compare sleep quality between the two waves of contagions and to examine the effect of resilience, together with sociodemographic and COVID-related variables, on sleep quality during the second wave.

Patients and Methods: A total of 648 participated in this longitudinal study through an online survey during the first lockdown consequent to the COVID-19 and during the second wave. The Medical Outcomes Study-Sleep Scale (MOS-SS) and the Resilience Scale (RS) were administered. Sociodemographic and COVID-related information were also collected. Results: The results showed sleep quality slightly increased in the second wave, even though with a small effect size. Correlational analysis showed that resilience is inversely correlated with sleep quality measured in the two waves. Sleep quality during the second wave was positively correlated with sleep quality in the first lockdown. Likewise, the results of multiple regression revealed that the sleep quality in the first lockdown and resilience were significant predictors of sleep quality during the second wave.

Conclusion: These findings highlighted that the prevalence of poor sleepers remained high during the second wave. Together with the sleep quality in the first lockdown, resilience represents an important factor related to sleep quality during the second wave. Interventions to improve sleep quality among the general population should take into account these findings.

Keywords: clinical psychology, health, sleep quality, resilience, longitudinal

\section{Introduction}

At the beginning of March 2020, while the World Health Organization declared the COVID-19 outbreak a pandemic, ${ }^{1}$ the Italian Government choose to adopt an extensive lockdown aiming to contain the contagion. As the epidemic advanced, a psychological impact on Italian people forced to stay at home with the fear of contagion was hypothesized.

Findings from the first studies have reported a heavy psychological impact among the Italian population. Approximately $20-30 \%$ of the people had moderate to extremely severe symptoms of depression, anxiety, and stress. ${ }^{2-5}$ Higher levels of anxiety and depression were associated with a wide array of maladaptive psychological strategies in healthy subjects as well as patients with pre-existing psychiatric problems $^{6}$ and less ability to activate family and friends' social support. ${ }^{7}$ 
During the most critical phase of the pandemic, people who experienced anxiety and depression are more likely to be absorbed in an inner fantasy world, which in turn may increase their emotional distress. ${ }^{8}$ Nonetheless, these were not the only forms of psychological symptoms Italian people experienced. A study involving a large sample of subjects has found that more than half of them reported poor sleep quality including sleep disturbance, awakening with shortness of breath or with headache, sleep inadequacy, and somnolence. 9 Along with changes in sleep, another study has well documented a higher frequency of nightmares during the Italian first lockdown in a large sample of 5988 adults. ${ }^{10}$

Sleep disturbances during the COVID-19 have received an increasing interest among researchers due to their relationship with other mental health disorders, ${ }^{11,12}$ and thereby, findings of these studies may be useful for prevention interventions. For example, together with an acceptable time for recreational activity, a good sleep quality seems to protect from posttraumatic stress disorder a professional category heavily under pressure during the pandemic as the healthcare workers. ${ }^{13}$ Put another way, sleep disturbances, on one hand, are related to the onset and worsening of psychiatric conditions and, on the other hand, represent the common consequence of exposure to traumatic events. ${ }^{14}$ Interestingly, a study has found a significant indirect effect from exposure level to symptoms of post-traumatic stress via sleep quality in a sample of Chinese healthcare workers facing the COVID-19 pandemic at peak of the contagion. ${ }^{15}$

Because of the high prevalence of sleep problems during the lockdown following the COVID-19 pandemic, a deeper understanding of the involved psychological processes is needed for preventing and treating people who are more vulnerable. No matter only how sociodemographic and COVID-related characteristics may be associated with sleep disturbances. Another issue of interest to researchers is indeed to identify the potential predictors of psychological symptoms and poor sleep quality. A crosssectional study showed that female gender, the death of a loved one for COVID-19, and extremely severe levels of depression, anxiety, and stress were significant predictors of poor sleep quality during the first lockdown. ${ }^{9}$ The role of some psychological factors historically associated with stress management was taken into account for understanding the individual psychological impact of the COVID-19 pandemic. For example, in one study, the authors found that insecure attachment style predicted symptoms of psychological distress in a large sample of Italian people. ${ }^{4}$ Another factor associated with how a subject cope with distress is psychological resilience. Research has documented the role of resilience in mitigating the psychological impact of the COVID-19 pandemic among the general population and healthcare workers. ${ }^{2,16}$ A further study showed the relation between resilience and ability to tolerate uncertainty as relevant factors to influence depersonalization in managing the impact of the COVID-19 emergency on health care workers. The use of emotional strategies that allow individuals to stay in a critical situation without the need to control it appears to protect against burnout in these circumstances. ${ }^{17}$

Likewise, a growing number of studies have showed the predictive or moderator role of resilience in sleep quality in the context of both non-clinical ${ }^{18-20}$ and psychiatric samples. ${ }^{21}$ Furthermore, resilience seems to buffer the potentially harmful effect of perceived stress in the onset of sleep disturbances. ${ }^{22}$ On the other hand, other studies have shown a relationship between insomnia/poor sleep and low level of resilience with the hypothesis that sleep disturbances may affect resilience and vice versa. ${ }^{23-25}$ Despite the straight relationship between sleep problems and resilience, there is a paucity of studies that have examined the association between poor sleep quality and resilience among the general population experiencing consequences of lockdown, such as orders to stay at home, be quarantined, and isolation from others.

The first aim of this study was to explore sleep quality between the first and the second wave. We hypothesized a poor and stable sleep quality during the second wave. The second aim of this study was to investigate the relationships between resilience, sleep quality during the first lockdown, and sleep quality during the second wave. We hypothesized that we would find a positive correlation between the two measurements of sleep quality. We also hypothesized that we would find an inverse relationship between resilience and sleep quality. The third aim of this study was to examine the effect of resilience, together with sociodemographic and COVID-related variables, on sleep quality during the second wave of contagions. We hypothesized that some of these variables and resilience would significantly impinge on poor sleep quality.

\section{Materials and Methods}

\section{Participants and Procedure}

This study is part of a larger and multicenter research project named "Resilience and the COVID-19: how to 
react to perceived stress. Effects on sleep quality and diurnal behavior/thoughts." A longitudinal design to assess sleep quality during the first lockdown and the second wave in Italy was adopted. A total of 6314 subjects took part in this study, an online survey system during the first Italian lockdown (T1: March 29-May 4). As shown in Figure 1, due to incomplete data or outlier identification, 659 cases were excluded and, consequently, the final sample at $\mathrm{T} 1$ consisted of 5655 subjects.

T1 measures were the Medical Outcomes StudySleep Scale (MOS-SS, Italian adaptation; ${ }^{26}$ ) and the Wagnild and Young Resilience Scale (RS). ${ }^{27,28}$ The T1 respondents were asked to participate in a second measurement (T2) on December 18, 2020. A total of 648 subjects (11.58\%) participated in the T2 measurement between December 18, 2020, and January 18, 2021. The MOS-SS was administered at the T2 measurement.

Inclusion criteria were an age of 18 years or over, living in Italy during the two waves of the COVID-19 pandemic, and an adequate understanding of the Italian language. Exclusion criteria were a pre-existing mental health disorder and/or taking psychotropic drugs. All participants completed the survey anonymously and gave informed consent electronically before participating. Privacy of the participants was guaranteed in accordance with the European Union General Data Protection Regulation 2016/679. This study was conducted in accordance with the 1964 Declaration of Helsinki and its later amendments. The study was approved by the Research Ethics Committee for Psychological Research of the University of Messina, Italy (no. 37442).

\section{Measures}

Sociodemographic and COVID-Related Information

Sociodemographic variables comprise age, gender, education, relationship status, and geographical area of residence. The COVID-related information included having contracted the virus, mandatory quarantine, infected acquaintances or loved ones in the last 3 months. The death of loved ones for COVID-19 was also collected.

\section{Sleep Quality: The Medical Outcomes Study-Sleep Scale}

The Medical Outcomes Study-Sleep Scale ${ }^{29}$ (MOS-SS) is a self-report instrument to assess sleep quality and quantity within a 4-week period. For the aims of this study, we only took into account the scores of the Sleep Index II assessing the quality of sleep. Based on the original validation study, indeed, the Sleep Index II represents the global index of MOS-SS. ${ }^{30}$ Scores range from " 0 " to " $100 "$, with higher scores suggesting greater sleep problems. Specifically, scores below 25.8 indicate greater sleep problems, as indicated by Hays and colleagues. ${ }^{29}$

In the current study, the Italian version of the MOS-SS showing excellent psychometric properties was used. ${ }^{26}$ Adequate level of reliability was detected in this sample, with a Cronbach's $\alpha$ of 0.083 for the Sleep Index II.

\section{Resilience: The Resilience Scale}

The Wagnild and Young Resilience Scale $^{27}$ (RS) is a selfreport instrument to measure resilience, which is defined as a personal and positive characteristic that enhances individual adaptation to adversity. It consists of 24 items rated on a 7-point Likert scale to "1" (disagree) to "7" (agree) that are grouped into five factors as follows: 1) meaningfulness, which measures the sense of having something for which live; 2) self-reliance, which measures the beliefs in oneself and one's abilities; 3) perseverance, which measures perseverance despite adversity or discouragement; 4) existential aloneness, which measures feeling of freedom and sense of uniqueness; and 5) equanimity, which measures a balanced perspective vision of one's life and experience.

In addition, an RS total score can be computed with scores of 126.6 and above denoting high resilience, as indicated by Girtler and colleagues. ${ }^{28}$ In the current study, a validated Italian version of the RS was adopted. $^{28}$ Several studies have highlighted that the RS is a simple and reliable tool with good psychometric properties. $^{2,27,28,31-33}$ In this study, an excellent degree of reliability was detected, with a Cronbach's $\alpha$ of 0.94 for the RS total score.

\section{Statistical Analysis}

The data were analyzed using SPSS v. 26 (IBM, Armonk, NY, USA) statistical software and Excel software v. 16.0 (Microsoft Corp. 2016). The prevalence of poor sleepers during the second wave was calculated according to the MOS-SS cut-off score indicated by Hays and colleagues: ${ }^{29}$ Sleep Index II $>25.8$. An independent $t$-test was used to compare the Sleep Index II score in the first and the second wave. The effect size (Cohen's d) was also calculated to quantify the difference between the two measurements. Relationships between the RS, the Sleep Index II in the first lockdown, and the Sleep Index II during the second wave were performed with Pearson 


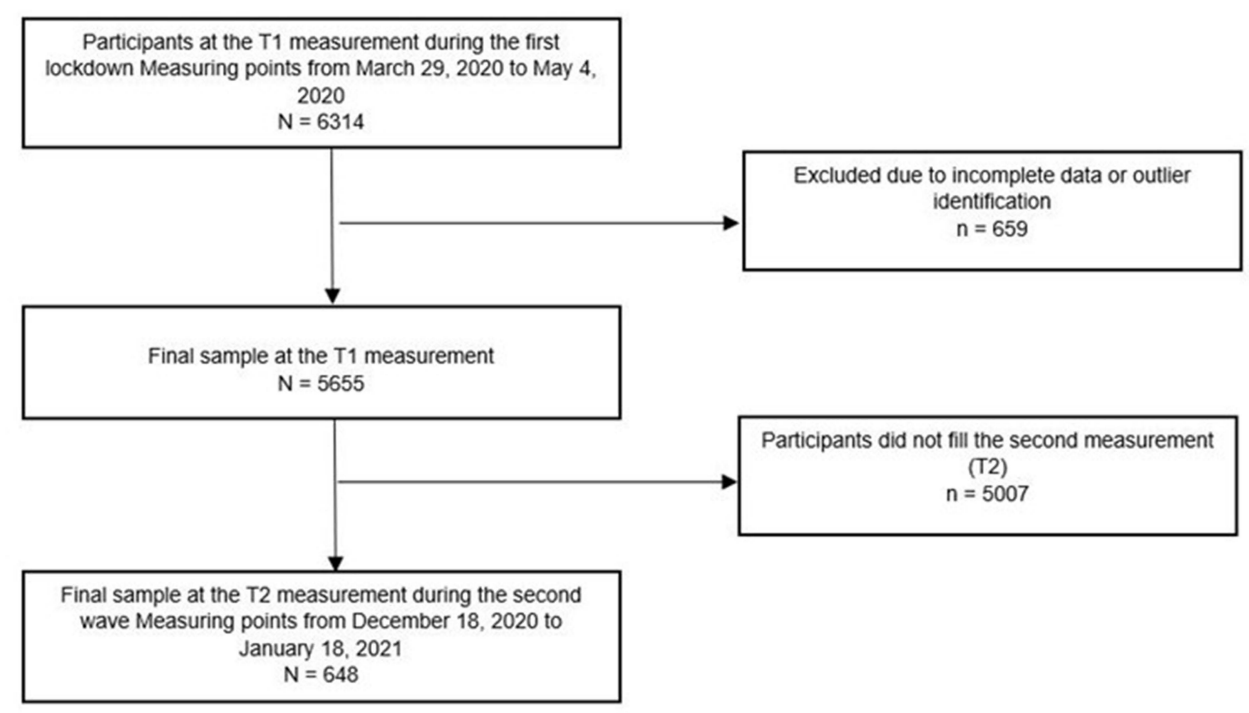

Figure I Flow chart of the study participants.

product-moment correlation coefficients. A hierarchical multiple regression analysis was performed to investigate the role of sociodemographic variables, COVID-related items, and resilience on the sleep quality during the second wave (T2). The independent variables were entered in four steps. The T2 Sleep Index II was the dependent variable, while age, gender, level of education, relationship status, and geographical area of residence were added as covariates in all four steps. In the second step, we inserted the T1 Sleep Index II. In the third step, we inserted the COVID-19-related variables as follows: COVID-19 infection in the last 3 months, mandatory quarantine for COVID-19 in the last 3 months, infected acquaintances or loved ones in the last 3 months, and death of loved ones for COVID-19. In the fourth step, we added the resilience total score.

We further carried out another hierarchical multiple regression analysis with the resilience factors (ie, meaningfulness, self-reliance, perseverance, existential aloneness, and equanimity) in the fourth step to explore which aspects of resilience are related to the dependent variable.

\section{Results}

\section{Sociodemographic and COVID-Related Information}

Table 1 shows sociodemographic and COVID-related information of the sample. The largest age range (42\%) was between 18 and 25 years of age $(M=32.92 ; S D=$ $13.01)$ and most of the participants were female (78.7\%) and were living in Northern Italy (77.3\%). Also, most of the participants had a high school diploma (46\%) and were married or in a steady relationship (62.3\%). Among participants, $5.2 \%$ had a COVID-19 infection in the last 3 months and $17 \%$ were in mandatory quarantine. About $48 \%$ of the participants stated that at least an acquaintance or loved one had been infected in the last 3 months. Finally, 9.6\% had a loved one who had died for COVID-19.

\section{Sleep Quality Differences Between the First (TI) and the Second Wave (T2)}

Figure 2 displays that the percentage of people who were poor sleepers $(\mathrm{T} 1$ prevalence $=66.4 \%)$ slightly decreased in the second wave (T2 prevalence $=62.7 \%$ ). Likewise, as shown in Table 2, the T1 Sleep Index II mean scores $(M=$ 33.28; $S D=13.02)$ were higher than the T2 Sleep Index II scores $(M=31.40 ; S D=11.91)$ and these differences were statistically significant $\left(t_{(1294)}=2.72 ; p<0.01\right)$, even though the effect size was small (Cohen's $d=0.15$ ).

\section{Correlational Analysis Between}

Resilience, Sleep Quality in the First Lockdown and Sleep Quality During the Second Wave

Table 2 shows descriptive statistics and correlation analyses. Results showed that the RS was inversely and weakly correlated with both the T1 Sleep Index II ( $r=$ 
Table I Sociodemographic and COVID-Related Information

\begin{tabular}{|l|l|l|}
\hline \multicolumn{2}{|l|}{ Overall Sample (N=648) } & $\%$ \\
\hline Age, years & 272 & 42 \\
\hline $18-25$ & 103 & 15.9 \\
\hline $26-30$ & 90 & 13.9 \\
\hline $31-40$ & 85 & 13.1 \\
\hline $41-50$ & 79 & 12.2 \\
\hline $51-60$ & 19 & 2.9 \\
\hline$>60$ & & 21.3 \\
\hline Gender & 138 & 78.7 \\
\hline Male & 510 & \\
\hline Female & \multicolumn{2}{|l}{} \\
\hline
\end{tabular}

Education level

\begin{tabular}{|l|l|l|}
\hline Primary or middle school & 16 & 2.5 \\
\hline High school diploma & 298 & 46 \\
\hline Graduate & 275 & 42.4 \\
\hline Postgraduate & 59 & 9.1 \\
\hline
\end{tabular}

Partnership

\begin{tabular}{|l|l|l|}
\hline Unmarried, divorced or widowed & 244 & 37.7 \\
\hline Married or in a steady relationship & 404 & 62.3 \\
\hline
\end{tabular}

Area of residence

\begin{tabular}{|l|l|l|}
\hline Northern Italy & 501 & 77.3 \\
\hline Central-southern Italy & 147 & 22.7 \\
\hline
\end{tabular}

COVID-19 infection in the last 3 months

\begin{tabular}{|l|l|l|}
\hline Yes & 36 & 5.6 \\
\hline No & 612 & 94.4 \\
\hline
\end{tabular}

Mandatory quarantine for COVID- 19 in the last 3 months

\begin{tabular}{|l|l|l|}
\hline Yes & 110 & 17 \\
\hline No & 538 & 83 \\
\hline
\end{tabular}

Infected acquaintances or loved ones in the last $\mathbf{3}$ months

\begin{tabular}{|l|l|l|}
\hline Yes & 308 & 47.5 \\
\hline No & 340 & 52.5 \\
\hline
\end{tabular}

Death of loved ones for COVID-19

\begin{tabular}{|l|l|l|}
\hline Yes & 62 & 9.6 \\
\hline No & 586 & 90.4 \\
\hline
\end{tabular}

$-0.15 ; p<0.01)$ and the T2 Sleep Index II $(r=-0.17 ; p<$ $0.01)$. Results of correlational analysis also showed that the T2 Sleep Index II was positively and highly correlated with the T1 Sleep Index II $(r=0.63 ; p<0.01)$.

\section{Predictors of Sleep Quality During the Second Wave (T2 Sleep Index II)}

Table 3 illustrates the results of hierarchical multiple regression on the T2 Sleep Index II as the outcome. Age $(\beta=$ $-0.13 ; p<0.01)$ and gender $(\beta=0.14 ; p<0.01)$ were statistically significant in step 1 , even though they did not reach the statistical significance after adding the T1 Sleep Index II in step 2. Indeed, results showed that the T1 Sleep Index II $(\beta=0.61 ; p<0.01)$ was the only predictor in step 2 . We found the same effect of the T1 Sleep Index II $(\beta=0.61$; $p<0.01)$ in step 3 , where we added the COVID-related variables. We also found a significant effect of having lost a loved one for COVID-19 in the last 3 months $(\beta=0.06$; $\mathrm{p}<0.05)$. Each of these steps reached a statistical significance. In step 4, the effect of the T1 Sleep Index II $(\beta=0.60$; $p<0.01)$ persisted and we also noted a significant effect of the resilience score $(\beta=-0.07 ; p<0.05)$. The final model explained about $42 \%$ of variance in the T2 Sleep quality index II $\left(R^{2}=0.415^{* *} ; p<0.01\right)$.

A further regression analysis was carried out to explore which aspects of resilience predict the sleep quality during the second wave. Because the first three steps were identical to the regression analysis using the total score of resilience, the results were not reported in the table Regarding the resilience factors added in the fourth step, we observed that only the Meaningfulness factor was a statistical predictor of the T2 Sleep Index II $(\beta=$ $-0.14 ; p<0.05)$.

\section{Discussion}

In the present study, we explored sleep quality throughout the course of the COVID-19 pandemic in a sample of Italian people. We collected longitudinal data from an online survey during the first wave and the consequent lockdown in spring 2020 (T1) as well as during the second wave, which begun in Autumn 2020 (T2). We also considered psychological resilience to explain sleep quality among participants.

Specifically, the first aim of this study was to verify sleep quality between the first and the second wave. In this 


\section{A T1 assement (from 29 March 2020 to 4 May 2020)}

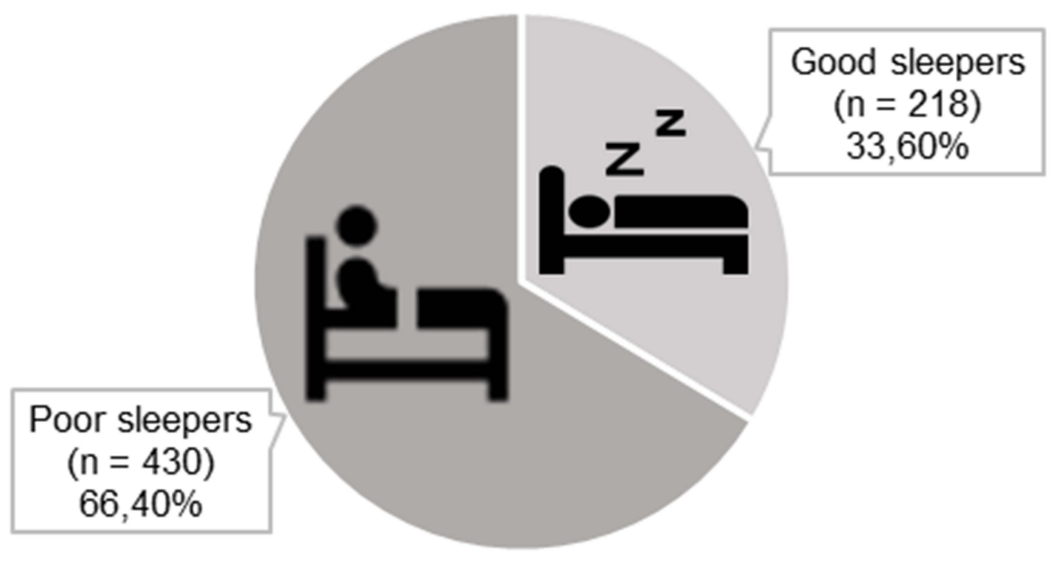

\section{B T2 assessment (from 18 December 2020 to 18 January 2021)}

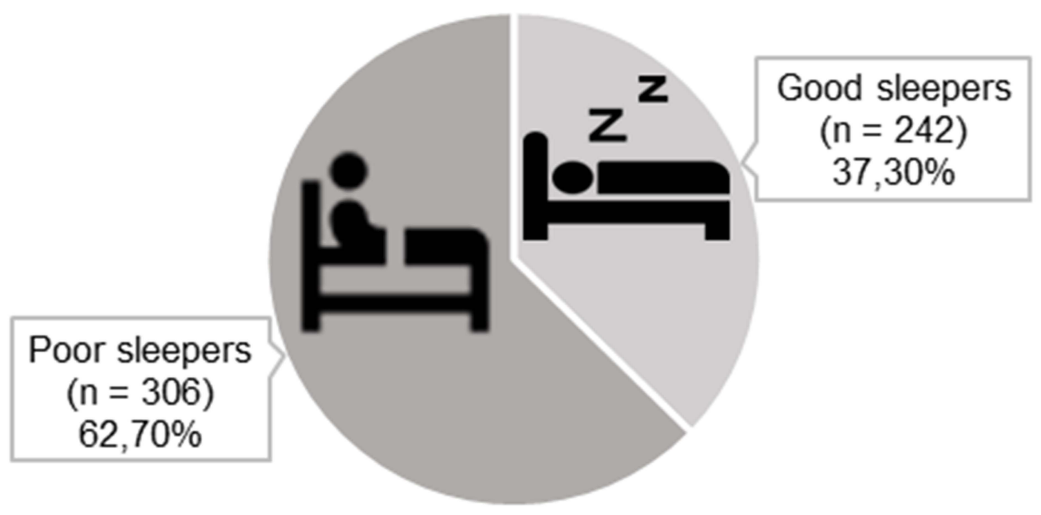

Figure 2 Percentages of poor sleepers and good sleepers during the TI and the T2 assessments.

Notes: A Sleep Index II score $>25.8$ was categorized as Poor sleeper; A Sleep Index II score $<25.8$ was categorized as Good sleeper. The icons are selected from the https:// icons8.com/icon/105962/subire-l'insonnia and https://icons8.com/icon/10787/dormire-nel-letto.

vein, we used the Medical Outcomes Study-Sleep Scale the second wave, from about $66 \%$ to $63 \%$, respectively. assessing sleep quality within a 4-week period. We found Descriptive results of sleep quality corroborated these that the prevalence of poor sleepers decreased during findings on the grounds that mean scores of the two

Table 2 Correlational Analysis Between TI RS, TI Sleep Index II, and T2 Sleep Index II

\begin{tabular}{|l|c|c|c|c|}
\hline & M & SD & I. TI Resilience Scale & 2. TI Sleep Index II \\
\hline I. TI Resilience Scale & 127.40 & 23.00 & & \\
\hline 2. TI Sleep Index II & 33.28 & 13.02 & $-0.15^{* *}$ & \\
\hline 3. T2 Sleep Index II & 31.40 & 11.91 & $-0.17^{* *}$ & $0.63^{* *}$ \\
\hline
\end{tabular}

Note: $* * p<0.01$. 
Table 3 The Regression Results of the Effects of Resilience on the T2 Sleep Index II

\begin{tabular}{|c|c|c|c|c|c|c|c|}
\hline $\begin{array}{l}\text { Predictor of the Sleep Quality During the Second } \\
\text { Wave }\end{array}$ & B & $\begin{array}{c}\text { B } \\
95 \% \mathrm{Cl} \\
{[\mathrm{LL}, \mathrm{UL}]}\end{array}$ & Beta & $s r^{2}$ & $R$ & Fit & Difference \\
\hline (Intercept) & $1.97 * *$ & {$[1.60,2.34]$} & & & & & \\
\hline Age & $-0.01 * *$ & {$[-0.01,-0.02]$} & -0.13 & -0.13 & -0.15 & & \\
\hline Gender & $0.24 * *$ & {$[0.11,0.38]$} & 0.14 & 0.14 & 0.16 & & \\
\hline Education level & -0.03 & {$[-0.09,0.02]$} & -0.05 & -0.05 & -0.06 & & \\
\hline Partnership & 0.08 & {$[-0.04,0.19]$} & 0.05 & 0.05 & 0.02 & & \\
\hline \multirow[t]{2}{*}{ Area of residence } & 0.06 & {$[-0.08,0.19]$} & 0.03 & 0.03 & 0.04 & & \\
\hline & & & & & & $R^{2}=0.046^{* *}$ & \\
\hline (Intercept) & $0.95 * *$ & {$[0.63,1.26]$} & & & & & \\
\hline Age & -0.01 & {$[-0.01,0.00]$} & -0.06 & -0.06 & -0.15 & & \\
\hline Gender & 0.09 & {$[-0.02,0.20]$} & 0.05 & 0.05 & 0.16 & & \\
\hline Education level & -0.01 & {$[-0.05,0.04]$} & -0.01 & -0.01 & -0.06 & & \\
\hline Partnership & 0.02 & {$[-0.07,0.1 \mathrm{I}]$} & 0.01 & 0.01 & 0.02 & & \\
\hline Area of residence & -0.06 & {$[-0.17,0.05]$} & -0.03 & -0.03 & 0.04 & & \\
\hline \multirow[t]{2}{*}{ T2 Sleep Index II } & $0.60 * *$ & {$[0.53,0.66]$} & 0.61 & 0.60 & 0.63 & & \\
\hline & & & & & & $R^{2}=0.402^{* *}$ & $\Delta R^{2}=0.356$ \\
\hline (Intercept) & $0.62^{*}$ & {$[0.21,1.02]$} & & & & & \\
\hline Age & -0.01 & {$[-0.01,0.00]$} & -0.06 & -0.05 & -0.15 & & \\
\hline Gender & 0.09 & {$[-0.02,0.20]$} & 0.05 & 0.05 & 0.16 & & \\
\hline Education level & -0.01 & {$[-0.05,0.03]$} & -0.01 & -0.01 & -0.06 & & \\
\hline Partnership & 0.01 & {$[-0.08,0.1 \mathrm{I}]$} & 0.01 & 0.01 & 0.02 & & \\
\hline Area of residence & -0.05 & {$[-0.16,0.06]$} & -0.03 & -0.03 & 0.04 & & \\
\hline T2 Sleep Index II & $0.60 * *$ & {$[0.54,0.66]$} & 0.61 & 0.60 & 0.63 & & \\
\hline COVID-19 infection in the last 3 months & 0.02 & {$[-0.21,0.24]$} & 0.01 & 0.01 & 0.06 & & \\
\hline Mandatory quarantine for COVID-19 in the last 3 months & 0.09 & {$[-0.05,0.23]$} & 0.05 & 0.04 & 0.08 & & \\
\hline Infected acquaintances or loved ones in the last 3 months & 0.03 & {$[-0.07,0.12]$} & 0.02 & 0.02 & 0.10 & & \\
\hline \multirow[t]{2}{*}{ Death of loved ones for COVID-19 } & $0.16 *$ & {$[0.01,0.31]$} & 0.06 & 0.06 & 0.06 & & \\
\hline & & & & & & $R^{2}=0.410^{* *}$ & $\Delta R^{2}=0.008$ \\
\hline (Intercept) & $0.88 * *$ & {$[0.42,1.35]$} & & & & & \\
\hline Age & -0.01 & {$[-0.01,0.01]$} & -0.05 & -0.05 & -0.15 & & \\
\hline Gender & 0.09 & {$[-0.02,0.20]$} & 0.05 & 0.05 & 0.16 & & \\
\hline Education level & -0.01 & {$[-0.05,0.03]$} & -0.02 & -0.02 & -0.06 & & \\
\hline
\end{tabular}


Table 3 (Continued).

\begin{tabular}{|c|c|c|c|c|c|c|c|}
\hline $\begin{array}{l}\text { Predictor of the Sleep Quality During the Second } \\
\text { Wave }\end{array}$ & B & $\begin{array}{c}\text { B } \\
95 \% \mathrm{Cl} \\
{[\mathrm{LL}, \mathrm{UL}]}\end{array}$ & Beta & $s r^{2}$ & $\boldsymbol{R}$ & Fit & Difference \\
\hline Partnership & 0.02 & {$[-0.08,0.11]$} & 0.01 & 0.01 & 0.02 & & \\
\hline Area of residence & -0.04 & {$[-0.14,0.07]$} & -0.02 & -0.02 & 0.04 & & \\
\hline T2 Sleep Index II & $0.59 * *$ & {$[0.52,0.65]$} & 0.60 & 0.58 & 0.63 & & \\
\hline COVID-19 infection in the last 3 months & 0.02 & {$[-0.20,0.25]$} & 0.01 & 0.01 & 0.06 & & \\
\hline Mandatory quarantine for COVID-19 in the last 3 months & 0.08 & {$[-0.06,0.22]$} & 0.04 & 0.04 & 0.08 & & \\
\hline Infected acquaintances or loved ones in the last 3 months & 0.03 & {$[-0.06,0.13]$} & 0.02 & 0.02 & 0.10 & & \\
\hline Death of loved ones for COVID-19 & 0.14 & {$[-0.01,0.30]$} & 0.06 & 0.06 & 0.06 & & \\
\hline \multirow[t]{2}{*}{ Resilience Scale total score } & $-0.01 *$ & {$[-0.02,-0.01]$} & -0.07 & -0.07 & -0.17 & & \\
\hline & & & & & & $R^{2}=0.415^{* *}$ & $\Delta R^{2}=0.004$ \\
\hline
\end{tabular}

Notes: A significant b-weight indicates the beta-weight and semi-partial correlations are also significant. $s r^{2}$ represents the semi-partial correlation squared. $r$ represents the zero-order correlation. $L L$ and $U L$ indicate the lower and upper limits of the confidence interval for $B . *_{p}<0.05$. $*_{p}^{*}<0.01$.

measurements (assessed by the Sleep Index II) were quite similar. Of particular interest is that the prevalence of poor sleepers in our sample was higher than findings from cross-sectional studies conducted during the first lockdown. Indeed, for example, Franceschini et $\mathrm{al}^{9}$ reported a prevalence of $55.32 \%$, coherent with about $52 \%$ and $57 \%$ of two other studies involving Italian participants. ${ }^{34,35}$ Moreover, although the improvement in sleep quality was statistically significant, we found only a small effect size. This result could in part depend on the heavy restrictive measures due to the quick increase in the number of infected starting in autumn 2020 and determining a protracted period of emergency. The stable and poor sleep quality across the measurements may also depend on the consequences of the first lockdown when the restrictive measures have involved the entire Italian national territory. Longitudinal studies focusing on sleep quality between the wave have outlined a similar picture. Salfi et $\mathrm{al}^{36}$ reported a negative and prolonged impact of the COVID-19 pandemic on sleep, with no differences between the first and the second wave. Analogous results were found by Conte et al. ${ }^{37}$ As in the previously cited study, they found that sleep quality reported by the participants was low and continued during the second wave. The prolonged lockdown and/or the uncertainty of the duration could be probably the cause of increased perceived stress.

The second aim of this study was to examine the relationships between resilience and sleep quality during the two waves of the COVID-19 pandemic. While on one hand, psychological resilience was inversely associated with depression, anxiety, and stress during the first Italian lockdown, ${ }^{2}$ on the other hand, the high prevalence of poor sleep quality emerging from cross-sectional studies $^{9,36,37}$ have caused concern among researchers, together with the need to understand the relationship with protective factors. First of all, as revealed by our results, sleep quality reported by the participants during the first lockdown was highly correlated with that of the second wave. It is, after all, another evidence that a poor sleep quality characterized the COVID-19 pandemic, even when the restrictive measures have changed. When considering studies adopting longitudinal design, findings showed analogous results. ${ }^{36,37}$ For the sake of clarity, it is relevant to recall that these studies had found persistently low levels of sleep quality. However, in our view, more attention to psychological factors potentially preventing mental health disorders in the aftermath of traumatic events, such as resilience, would constitute a useful tool for a preventive approach. ${ }^{38}$ Unfortunately, very few studies have examined the interplay between sleep and resilience during the COVID-19 pandemic. $^{39}$ Our findings pointed out that resilience was correlated, even though weakly, with both the measurements of sleep quality. Therefore, people with higher resilience scores had a better sleep quality not only in the first lockdown but even during the second wave of contagion. In 
light of this finding, resilience can help grasp why some individuals experience poor sleep quality, while others do not. Nonetheless, other variables, such as sociodemographic and COVID-related characteristics of the subjects, may be responsible for the low correlation coefficients we found.

The final aim of this study was to explore the effect of resilience on sleep quality among participants during the second wave and the related restrictive measures. To date, there is no shortage of evidence linked to the psychological impact of the COVID-19 pandemic, ${ }^{40}$ even though the long-term effects on the general population are still unknown. Because past studies during the first wave have widely used a cross-sectional design that did not allow us to clarify the causal pathways from psychological functioning to psychopathology, we adopted a longitudinal design to examine the predictive role of resilience on sleep quality. In the hierarchical regression model, we also took into account the sleep quality reported by participants during the first lockdown, together with sociodemographic information and COVID-related variables within the last 3 months. As pointed out by our results, age and gender were significant predictors in the first step of the regression model, even though they explained only a small percentage of the variance (about $4 \%$ ). Perhaps not surprisingly then, the greatest contribution is given by the sleep quality measured during the first lockdown, while socio-demographic characteristics did not reach a statistical significance in the subsequent steps of the regression analysis. Accordingly, our results are different from those of Salfi et al. ${ }^{36}$ Findings of their study revealed that being older and having a relative or friend infected in the last 2 weeks represented risk categories for poor sleep quality. However, these differences may depend on the variables considered insofar they inserted as predictors in the regression model only sociodemographic and COVID19-related variables. At the same time, our main interest was to verify the effect of resilience on sleep quality. In our opinion, the identification of psychological factors that can reduce the psychological impact of the COVID-19 pandemic on the population is paramount. Recent studies have already demonstrated how resilience mitigates depression, sleep, and anxiety during the first lockdown among the general population and healthcare workers. $^{2,16}$ Our findings give evidence to the fact that resilience and sleep are strictly related. Thus, these data are coherent with other studies confirming the hypothesis of a relationship between sleep disturbances and low resilience. $^{23-25}$ Although poor sleep quality is one common consequence of the ongoing pandemic, our findings show that individual responses vary among people and may in part hinge on resilience.

We also explored which resilience factors are related to sleep quality during the second wave. Even if these findings can be viewed only as exploratory, we consider that can increase our understanding of the role playing by resilience. As can be evidenced by our results, the Meaningfulness factor was a significant predictor of the sleep quality reported by the participants during the second wave. Put another way, sleep quality may depend on the degree to which an individual holds a sense of having something to live for. Seemingly, meaningfulness seems to play a relevant role in explaining depression and stress during the early period characterized by the quick spread of the contagion in Italy. ${ }^{2}$ Whatever the cause, future research should take into account the differential role of the resilience factors in explaining sleep quality.

It is worth emphasizing that our findings may engender some practical implications for counteracting the psychological impact of the COVID-19 pandemic. Starting from these findings, together with the poor and stable sleep quality during the second wave emerging from research, prevention interventions centered on resilience could be particularly worthwhile to mitigate the consequences among the population. Likewise, the identification of high-risk subjects can be useful to avoid that poor sleep quality becoming chronic. Simple and reliable tools to evaluate the subjective perception of sleep quality, together with resilience, may be useful for this purpose. The current study has some limitations that should be considered in understanding the results. First, this study adopted a convenience sample and, so, the results may not be fully generalizable due to the oversampling of some characteristics, such as gender. Second, data were collected via an online survey that did not fully allow assessment for preexisting psychiatric disorders. However, this was the only way to collect data during the lockdown and its related restrictive measures. Third, the use of self-report measures of sleep quality did not allow to exclude a certain risk of response bias. Despite both the MOS-SS and the RS are reliable and commonly used instruments, social desirability could influence the answer given by the participants. 


\section{Conclusion}

In conclusion, our results pointed out a slight improvement in the quality of sleep from the first to the second wave of contagion. However, the sleep quality remained constantly poor among the participants. The results also emphasized that resilience assessed during the first wave was a predictor for sleep quality during the second wave of the COVID-19 pandemic. Together with resilience, the sleep quality measured in the first months of the pandemic represents a crucial predictor of sleep quality during the second wave of contagion. Prevention interventions focusing on resilience can decrease the impact of pandemic on sleep quality.

\section{Data Sharing Statement}

The data that support the findings of this study are available from the corresponding author on reasonable request.

\section{Ethics Approval and Informed Consent}

This study was conducted in accordance with the 1964 Declaration of Helsinki and its later amendments. The study was approved by the Research Ethics Committee for Psychological Research of the University of Messina, Italy (n. 37442). All participants completed the survey anonymously and gave informed consent electronically before participate.

\section{Funding}

There is no funding to report.

\section{Disclosure}

GP participated in advisory board for UCB Pharma, JazzPharmaceuticals, Bioproject, Takeda, and Idorsia. The remaining authors declare that the research was conducted in the absence of any commercial or financial relationships that could be construed as a potential conflict of interest.

\section{References}

1. World Health Organization. Coronavirus Disease 2019 (COVID-19). Situation Report - 116. Geneva: World Health Organization; 2020.

2. Lenzo V, Quattropani MC, Musetti A, et al. Resilience contributes to low emotional impact of the COVID-19 outbreak among the general population in Italy. Front Psychol. 2020;11:576485. doi:10.3389/ fpsyg.2020.576485

3. Mazza C, Ricci E, Biondi S, et al. A nationwide survey of psychological distress among Italian people during the COVID-19 pandemic: immediate psychological responses and associated factors. Int J Environ Res Public Health. 2020;17(9):3165. doi:10.3390/ ijerph17093165
4. Moccia L, Janiri D, Pepe M, et al. Affective temperament, attachment style, and the psychological impact of the COVID-19 outbreak: an early report on the Italian general population. Brain Behav Immun. 2020;87:75-79. doi:10.1016/j.bbi.2020.04.048

5. Schimmenti A, Starcevic V, Giardina A, Khazaal Y, Billieux J. Multidimensional assessment of COVID-19-related fears (MAC-RF): a theory-based instrument for the assessment of clinically relevant fears during pandemics. Front Psychiatry. 2020;11:748. doi:10.3389/fpsyt.2020.00748

6. Pfefferbaum B, North CS. Mental health and the Covid-19 pandemic. N Engl J Med. 2020;383(6):510-512. doi:10.1056/NEJMp2008017

7. Mariani R, Renzi A, Di Trani M, Trabucchi G, Danskin K, Tambelli R. The impact of coping strategies and perceived family support on depressive and anxious symptomatology during the coronavirus pandemic (COVID-19) lockdown. Front Psychiatry. 2020;11:587724. doi:10.3389/fpsyt.2020.587724

8. Musetti A, Franceschini C, Pingani L, et al. Maladaptive daydreaming in an adult Italian population during the COVID-19 lockdown. Front Psychol. 2021;12:631979. doi:10.3389/ fpsyg.2021.631979

9. Franceschini C, Musetti A, Zenesini C, et al. Poor sleep quality and its consequences on mental health during the COVID-19 lockdown in Italy. Front Psychol. 2020;11:574475. doi:10.3389/fpsyg.2020.5 74475

10. Scarpelli S, Alfonsi V, Mangiaruga A, et al. Pandemic nightmares: effects on dream activity of the COVID-19 lockdown in Italy. $J$ Sleep Res. 2021;30(5):e13300. doi:10.1111/jsr.13300

11. Freeman D, Sheaves B, Waite F, Harvey AG, Harrison PJ. Sleep disturbance and psychiatric disorders. Lancet Psychiatry. 2020;7 (7):628-637. doi:10.1016/S2215-0366(20)30136-X

12. Davies G, Haddock G, Yung AR, Mulligan LD, Kyle SD. A systematic review of the nature and correlates of sleep disturbance in early psychosis. Sleep Med Rev. 2017;31:25-38. doi:10.1016/j. smrv.2016.01.001

13. Geng S, Zhou Y, Zhang W, et al. The influence of risk perception for COVID-19 pandemic on posttraumatic stress disorder in healthcare workers: a survey from four designated hospitals. Clin Psychol Psychother. 2021;28(5):1146-1159. doi:10.1002/cpp.2564

14. Pickett SM, Barbaro N, Mello D. The relationship between subjective sleep disturbance, sleep quality, and emotion regulation difficulties in a sample of college students reporting trauma exposure. Psychol Trauma. 2016;8(1):25-33. doi:10.1037/tra0000064

15. Yin Q, Sun Z, Liu T, et al. Posttraumatic stress symptoms of health care workers during the Corona virus disease 2019. Clin Psychol Psychother. 2020;27(3):1-12. doi:10.1002/cpp.2477

16. Lenzo V, Mc Q, Sardella A, Martino G, Ga B. Depression, anxiety, and stress among healthcare workers during the COVID-19 outbreak and relationships with expressive flexibility and context sensitivity. Front Psychol. 2021;12:623033. doi:10.3389/fpsyg.2021.623033

17. Di Trani M, Mariani R, Ferri R, De Berardinis D, Frigo MG. From resilience to burnout in healthcare workers during the COVID-19 Emergency: the role of the ability to tolerate uncertainty. Front Psychol. 2021;12:646435. doi:10.3389/fpsyg.2021.646435

18. Du C, Zan MCH, Cho MJ, et al. Increased resilience weakens the relationship between perceived stress and anxiety on sleep quality: a moderated mediation analysis of higher education students from 7 countries. Clocks Sleep. 2020;2(3):334-353. doi:10.3390/clockssleep 2030025

19. Hrozanova M, Moen F, Pallesen S. Unique predictors of sleep quality in junior athletes: the protective function of mental resilience, and the detrimental impact of sex, worry and perceived stress. Front Psychol. 2019;10:1256. doi:10.3389/fpsyg.2019.01256

20. Li Y, Gu S, Wang Z, et al. Relationship between stressful life events and sleep quality: rumination as a mediator and resilience as a moderator. Front Psychiatry. 2019;10:348. doi:10.3389/ fpsyt.2019.00348 
21. Liu L, Perceived Stress CQ. Sleep quality among Chinese drug users: analysis of rumination as a mediator and resilience as a moderator Int J Ment Health Addict. 2020. doi:10.1007/s11469-020-00388-9

22. Liu X, Liu C, Tian X, et al. Associations of perceived stress, resilience and social support with sleep disturbance among community-dwelling adults. Stress Health. 2016;32(5):578-586. doi:10.1002/smi.2664

23. Cheng P, Casement MD, Drake CL. Digital cognitive behavioral therapy for insomnia promotes later health resilience during the coronavirus disease 19 (COVID-19) pandemic. Sleep. 2021;44(4): zsaa258. doi:10.1093/sleep/zsaa258

24. Germain A, Dretsch M. Sleep and resilience-a call for prevention and intervention. Sleep. 2016;39(5):963-965. doi:10.5665/sleep.5732

25. Palagini L, Moretto U, Novi M, et al. Lack of resilience is related to stress-related sleep reactivity, hyperarousal, and emotion dysregulation in insomnia disorder. J Clin Sleep Med. 2018;14(5):759-766. doi: $10.5664 / \mathrm{jcsm} .7100$

26. Palagini L, Manni R. Misurare il sonno: Repertorio delle scale di valutazione dei disturbi del sonno. [Sleep Measurement: Directory of Sleep Disturbance Assessment Scales]. Minerva Medica; 2016:3-4.

27. Wagnild GM, Young HM. Development and psychometric evaluation of the Resilience Scale. J Nurs Meas. 1993;1(2):165-178.

28. Girtler N, Casari EF, Brugnolo A, et al. Italian validation of the Wagnild and Young Resilience Scale: a perspective to rheumatic diseases. Clin Exp Rheumatol. 2010;28(5):669-678.

29. Hays RD, Martin SA, Sesti AM, Spritzer KL. Psychometric properties of the Medical Outcomes Study Sleep measure. Sleep Med. 2005;6(1):41-44. doi:10.1016/j.sleep.2004.07.006

30. Hays RD, Stewart AL. Sleep measures. In: Stewart AL, Ware JE, editors. Measuring Functioning and Well-Being: The Medical Outcomes Study Approach. Durham, NC: Duke University Press; 1992:235-259.

31. Aroian KJ, Schappler-Morris N, Neary S, Spitzer A, Tran TV. Psychometric evaluation of the Russian language version of the resilience scale. J Nurs Meas. 1997;5(2):151-164. doi:10.1891/ $1061-3749.5 .2 .151$
32. Heilemann MV, Lee K, Kury FS. Psychometric properties of the Spanish version of the resilience scale. J Nurs Meas. 2003;11 (1):61-72. doi:10.1891/jnum.11.1.61.52067

33. Lundman B, Strandberg G, Eisemann M, Gustafson Y, Brulin C. Psychometric properties of the Swedish version of the resilience scale. Scand J Caring Sci. 2007;21(2):229-237. doi:10.1111/j.14716712.2007.00461.x

34. Casagrande M, Favieri F, Tambelli R, Forte G. The enemy who sealed the world: effects quarantine due to the COVID-19 on sleep quality, anxiety, and psychological distress in the Italian population. Sleep Med. 2020;75:12-20. doi:10.1016/j.sleep.2020.05.011

35. Cellini N, Canale N, Mioni G, Costa S. Changes in sleep pattern, sense of time and digital media use during COVID-19 lockdown in Italy. J Sleep Res. 2020;29(4):e13074. doi:10.1111/jsr.13074

36. Salfi F, D'Atri A, Tempesta D, Ferrara M. Sleeping under the waves: a longitudinal study across the contagion peaks of the COVID-19 pandemic in Italy. $J$ Sleep Res. 2021;30(5):e13313. doi:10.1111/ jsr. 13313

37. Conte F, Cellini N, De Rosa O, et al. Dissociated profiles of sleep timing and sleep quality changes across the first and second wave of the COVID-19 pandemic [published online ahead of print, $2021 \mathrm{Sep}$ 3]. J Psychiatr Res. 2021;143:222-229. doi:10.1016/j.jpsychires. 2021.09.025

38. Chen S, Bonanno GA. Psychological adjustment during the global outbreak of COVID-19: a resilience perspective. Psychol Trauma. 2020;12(S1):S51-S54. doi:10.1037/tra0000685

39. Cloonan SA, Taylor EC, Persich MR, Dailey NS, Killgore WDS. Sleep and resilience during the COVID-19 pandemic. In: Anxiety, Uncertainty, and Resilience During the Pandemic Period Anthropological and Psychological Perspectives; 2021. doi:10.5772/ intechopen. 98298

40. Xiong J, Lipsitz O, Nasri F, et al. Impact of COVID-19 pandemic on mental health in the general population: a systematic review. $J$ Affect Disord. 2020;277:55-64. doi:10.1016/j.jad.2020.08.001

\section{Publish your work in this journal}

Nature and Science of Sleep is an international, peer-reviewed, open access journal covering all aspects of sleep science and sleep medicine, including the neurophysiology and functions of sleep, the genetics of sleep, sleep and society, biological rhythms, dreaming, sleep disorders and therapy, and strategies to optimize healthy sleep.
The manuscript management system is completely online and includes a very quick and fair peer-review system, which is all easy to use. Visit http://www.dovepress.com/testimonials.php to read real quotes from published authors. 